Artículos Científicos

\title{
Impacto de la Experiencia de Compra Online en el Engagement Online del Consumidor del Sector Moda de la Ciudad de Aguascalientes
}

Impact of the Online Shopping Experience on the Online Engagement of the Consumer in the Fashion Industry in the City of Aguascalientes

Impacto da Experiência de Compras Online no Engajamento Online do Consumidor no Setor de Moda da Cidade de Aguascalientes

Vianney Judith Robledo Herrera Universidad Autónoma de Aguascalientes, México vianney.robledo@edu.uaa.mx https://orcid.org/0000-0003-1084-7507

Elena Patricia Mojica Carrillo Universidad Autónoma de Aguascalientes, México epmojica@correo.uaa.mx https://orcid.org/0000-0003-0112-4008

Sofía Elba Vázquez Herrera Universidad Autónoma de Aguascalientes, México sofiaelba.vazquez@upaep.mx https://orcid.org/0000-0001-8053-5678

Fernando Rey Castillo Villar Universidad Autónoma de Aguascalientes, México fernandorey.castillo@upaep.mx https://orcid.org/0000-0003-2011-8957 


\section{Resumen}

La presente investigación tuvo como objetivo general estudiar la relación entre la experiencia de compra online y el engagement online del consumidor del sector moda de la ciudad de Aguascalientes. Primero se presentan los aspectos más relevantes encontrados en la revisión de la literatura teórico-empírica. Y posteriormente se plantea, mediante el diseño de un modelo teórico, la posible relación directa y positiva entre las siguientes variables: experiencia de compra online y engagement online. El tipo de investigación que se realizó fue no experimental, de corte transversal y cuyo alcance fue correlacional-causal, lo que llevó a desarrollar un estudio empírico. La aplicación de un cuestionario a 440 jóvenes de 20 a 34 años de edad permitió obtener datos cuantitativos que fueron analizados con el software SPSS y modelizados a través de la técnica de ecuaciones estructurales para efectuar la contrastación de las hipótesis de investigación. Los resultados obtenidos establecieron una influencia directa y positiva de la experiencia de compra online sobre el engagement online. En otras palabras, la experiencia de compra online generada por los sitios web explica el engagement online de los consumidores del sector moda de la ciudad de Aguascalientes.

Palabras claves: e-commerce, engagement online, experiencia de compra online, marketing digital, moda.

\section{Abstract}

The general objective of this research was to study the relationship between the online shopping experience and the online engagement in the fashion sector of the city of Aguascalientes, Mexico. First, the most relevant aspects found in the review of the theoretical-empirical literature are presented. And later, through the design of a theoretical model, the possible direct and positive relationship between the following variables is raised: online shopping experience and online engagement. The type of research carried out was non-experimental, cross-sectional and whose scope was correlational-causal, which led to the development of an empirical study. The application of a questionnaire to 440 young people between 20 and 34 years of age allowed obtaining quantitative data that were analyzed with the SPSS software and modelled through the technique of structural equations to carry out the contrasting of the research hypotheses. The results established a direct and positive influence of the online shopping experience on online engagement. In other words, the online 


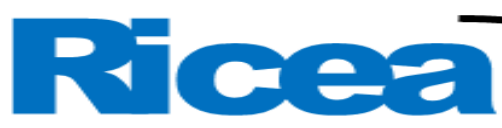

REVISTA IBEROAMERICANA DE CONTADURÍA, ECONOMÍA Y ADMINISTRACIÓN

ISSN $=2007-9907$

shopping experience generated by the websites explains the online engagement of consumers in the fashion sector of the city of Aguascalientes.

Keywords: e-commerce, online engagement, online shopping experience, digital marketing, fashion.

\section{Resumo}

O objetivo geral desta pesquisa foi estudar a relação entre a experiência de compra online e o engajamento online do consumidor no setor de moda da cidade de Aguascalientes. Primeiramente, são apresentados os aspectos mais relevantes encontrados na revisão da literatura teórico-empírica. E posteriormente, através do desenho de um modelo teórico, levanta-se a possível relação direta e positiva entre as seguintes variáveis: experiência de compra online e engajamento online. O tipo de investigação realizada foi não experimental, transversal e de âmbito correlacional-causal, o que conduziu ao desenvolvimento de um estudo empírico. A aplicação de um questionário a 440 jovens entre 20 e 34 anos permitiu a obtenção de dados quantitativos que foram analisados com o software SPSS e modelados por meio da técnica de equações estruturais para realizar o contraste das hipóteses de pesquisa. Os resultados obtidos estabeleceram uma influência direta e positiva da experiência de compra online no engajamento online. Em outras palavras, a experiência de compra online gerada pelos sites explica o engajamento online dos consumidores do setor de moda da cidade de Aguascalientes.

Palavras-chave: e-commerce, engajamento online, experiência de compra online, marketing digital, moda.

Fecha Recepción: Diciembre 2019 Fecha Aceptación: Junio 2020 


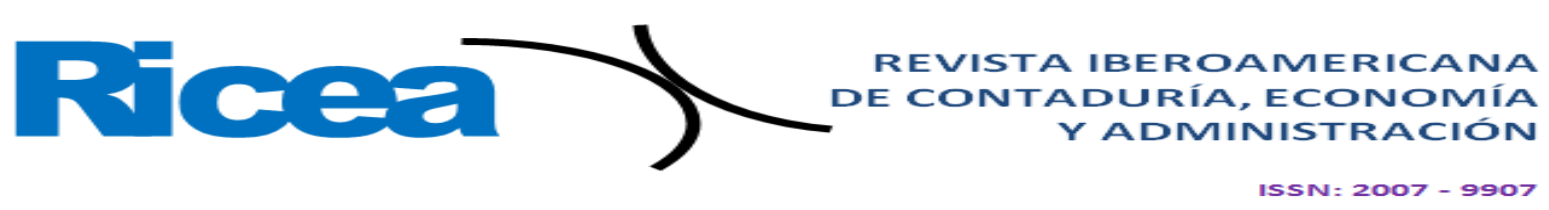

\section{Introducción}

El crecimiento global del comercio electrónico en los últimos años ha sido exponencial. Irala ( 2 de enero de 2018) menciona que las compras online se están volviendo una práctica habitual y con una mayor penetración en la población mundial. En el mundo existen aproximadamente 1600 millones de usuarios que han realizado compras en Internet y han invertido casi 2 billones de dólares, cifra que se estima podría duplicarse para este año 2020 (Irala, 2 de enero de 2018).

Particularmente, la evolución del comercio electrónico en México también ha ido en ascenso. De acuerdo con lo establecido en el Estudio sobre la Experiencia del Comprador 2017, elaborado por comScore (2017), se alcanzó la cifra de 17.63 billones de dólares en el 2016. Además, al mes de septiembre de 2017, $75 \%$ de los internautas mexicanos habían realizado una compra a través de Internet en los últimos tres meses, lo que representa un crecimiento de $4 \%$ con respecto al año anterior, según la misma fuente.

Es importante precisar que, siguiendo a comScore (2017), $40 \%$ de los productos ofertados en línea corresponden a la categoría de ropa y accesorios. Asimismo, la región Centro, a la que pertenece el estado de Aguascalientes, es la que más se desarrolló en el sector fashion (32\%), de acuerdo con la Asociación Mexicana de Venta Online [AMVO], Elogia y Netquest (2018). Desde el año 2016 a la fecha, ha sido la categoría que más adquiere el consumidor en Internet respecto al total de las categorías (59\%), seguida de las descargas digitales (48\%) y la compra de boletos para eventos (de $36 \%$ ) (comScore, 2017). En esa misma línea, esta categoría de ropa y accesorios registró un aumento en la incidencia de compra online en el año 2017 con respecto al 2016 de $6 \%$ (comScore, 2017). En promedio, los compradores gastan \$1699 para adquirir artículos de moda (AMVO, Elogia y Netquest, 2018).

Pero no solo en el entorno online tiene importancia este sector. Según el Instituto Nacional de Estadística y Geografía [Inegi] (2018), a nivel nacional la industria textil y de confección es una de las más importantes, ya que aporta 2.7 \% al producto interno bruto (PIB) y produce $10.3 \%$ del PIB en el sector manufacturero. Así pues, dado que contribuye en gran medida a la economía y a la expansión del comercio electrónico en México y particularmente en Aguascalientes, debido a que es la categoría en la que el consumidor invierte con más frecuencia en línea, lo cual atrae a los inversionistas y esto, a su vez, favorece el incremento de la competencia, el sector moda cobra una relevancia pocas veces dimensionada. 


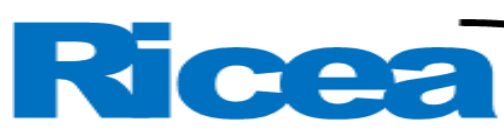

REVISTA IBEROAMERICANA DE CONTADURÍA, ECONOMÍA Y ADMINISTRACIÓN

Lo anterior a pesar de que es notable el reto que representa para las marcas de moda el comercializar sus productos en un entorno online, por tratarse de productos con un valor hedónico, cuyas características son simbólicas y experienciales (López, 2017), lo que hace que la optimización de la experiencia del usuario deba manejarse como un elemento estratégico de gran importancia (Sádaba, 2015, citado en López, 2017), debido a la necesidad que posee el consumidor de tener un contacto físico con el producto de cara a la decisión de compra (Blázquez, 2014).

Dado el contexto tecnológico antes mencionado, de acuerdo con López (2017), el entorno competitivo y globalizado en el que están inmersas las empresas ha dificultado desarrollar estrategias de negocio que garanticen su éxito y permanencia en el mercado a largo plazo.

En este sentido, estratégicamente una experiencia de compra online debe procurar una utilidad y facilidad de uso del sitio web, pero también favorecer el placer y entretenimiento de los consumidores durante el proceso de compra (Koufaris, 2002). De tal manera que ambos factores mejoren su percepción de la experiencia total y, como consecuencia, se incremente la probabilidad de compra y el gasto, así como la recomendación a familiares y amigos (Grewal, Levy y Kumar, 2009). Y aún más: se desencadene un mayor compromiso a largo plazo por parte del cliente (López, 2017).

A partir de lo anterior, la presente investigación se enfoca en comprender a profundidad el comportamiento del consumidor online a través de un estudio empírico. El objetivo es estudiar la relación entre la experiencia de compra online y el engagement online del consumidor del sector moda de la ciudad de Aguascalientes. Las variables que integran el modelo teórico propuesto son: experiencia de compra online y engagement online. En dicho modelo, se establecen las posibles relaciones entre estas variables, las cuales son sustentadas por la literatura científica consultada.

Por lo tanto, con base en el objetivo planteado para la realización de la presente investigación, se buscó responder la siguiente pregunta:

- ¿Cómo se relacionan la experiencia de compra online y el engagement online del consumidor del sector moda de la ciudad de Aguascalientes? 


\section{Marco teórico}

La literatura científica consultada indica que la experiencia de compra online es tratada desde varios puntos de vista, lo que la hace inconsistente en términos de su definición y explicación del concepto (Rose, Clark, Samouel y Hair, 2012). Para efectos de la presente investigación, se adoptará como definición de experiencia de compra online la propuesta por Trevinal y Stenger (2014), quienes afirman que es un proceso holístico y subjetivo que resulta de las interacciones entre los propios consumidores y el entorno online (sitios web de comercio electrónico, opiniones o valoraciones de productos de otros usuarios, redes sociales, etc.).

Como complemento a la definición antes mencionada, Rose et al. (2012) propone diez variables antecedentes, a saber: habilidad, nivel de desafío, telepresencia, velocidad interactiva, control percibido, conectividad, customización, facilidad de uso, estética y beneficios percibidos. Estas variables, a su vez, son formativas de dos componentes o estados de la experiencia de compra online: estado experiencial cognitivo y estado experiencial afectivo.

Por otra parte, el engagement es un concepto relativamente nuevo en el campo del marketing (Brodie, Ilic, Juric y Hollebeek, 2013). Si bien se comenzó a estudiar a partir del año 2005 con algunos trabajos como el de Patterson, Yu y de Ruyter (2006), quienes estudiaron el engagement del consumidor en el ámbito de los servicios, hasta el momento no hay una amplia base teórica. Precisamente, Vivek, Beatty y Morgan (2012) y Brodie et al. (2013) destacan que a pesar del amplio uso de este término relacionado con las marcas, la revisión de la literatura académica revela que no existe un consenso sobre su naturaleza semántica, así como tampoco lo hay de sus dimensiones.

Como sea que fuere, para efectos de la presente investigación, el engagement online es caracterizado como un vehículo para la creación y el mantenimiento de las relaciones entre el consumidor y la empresa. Se trata de un estado psicológico que sucede bajo ciertas condiciones que dependen del contexto en el que se desarrollan las experiencias interactivas y cocreativas de los consumidores con una marca, ya sea en un sitio web u otras entidades mediadas por computadora. Y genera diferentes niveles de compromiso dentro de las relaciones de servicio que crean valor más allá de la compra (Brodie, Hollebeek, Juric e Ilic, 2011; Hollebeek, 2011; Mollen y Wilson, 2010; Patterson et al., 2006; Van Doorn et al., 2010; Vivek et al.,2012). 


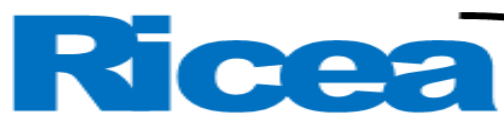

REVISTA IBEROAMERICANA DE CONTADURÍA, ECONOMÍA

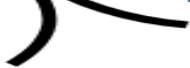

Y ADMINISTRACIÓN

ISSN $=2007-9907$

Así, de acuerdo con Calder, Malthouse y Schaedel (2009), el engagement online es un constructo de segundo orden compuesto por dos de primer orden: personal y socialinteractivo; y multidimensional: estimulación e inspiración, facilitación social, temporal, autoestima y civismo, disfrute intrínseco, utilitario, participación y socialización y comunidad.

\section{Materiales y métodos}

El proceso metodológico utilizado parte de la revisión de la literatura científica existente con el objetivo de identificar el problema de investigación y plantear los objetivos e hipótesis a contrastar, lo que condujo al planteamiento del modelo teórico en el que se centra la presente investigación.

A partir de la revisión de la literatura de estudios teórico-empírico se planteó la siguiente hipótesis:

$\mathrm{H}_{1}$ : La experiencia de compra online influye de manera directa y positiva en el engagement online del consumidor del sector moda de la ciudad de Aguascalientes.

Como resultado, se propone un modelo teórico que orienta la presente investigación y plantea de manera gráfica la posible relación entre las variables estudiadas. Dicho modelo deberá contrastarse empíricamente (figura 1).

Figura 1. Modelo general de la investigación

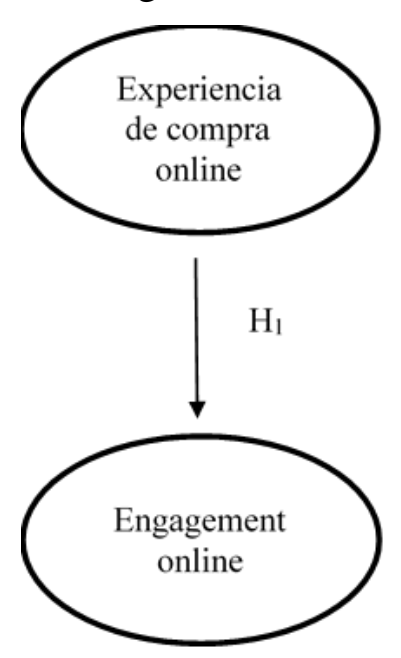

Fuente: Elaboración propia 


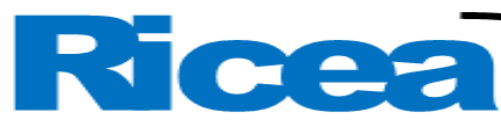

REVISTA IBEROAMERICANA DE CONTADURÍA, ECONOMÍA

En la tabla 1 se muestra la información técnica de la investigación empírica, mientras que en la tabla 2 y 3 se presentan los ítems por variable que fueron utilizados en el cuestionario aplicado.

Tabla 1. Ficha técnica de la investigación

\begin{tabular}{|c|c|}
\hline $\begin{array}{l}\text { Naturaleza de la } \\
\text { investigación }\end{array}$ & Cuantitativa correlacional-causal y transversal. \\
\hline Técnica de recolección & $\begin{array}{l}\text { Encuesta electrónica con aplicación de cuestionario } \\
\text { estructurado cuyo muestreo fue por bola de nieve. }\end{array}$ \\
\hline Ámbito geográfico & Ciudad de Aguascalientes \\
\hline Población & $\begin{array}{l}1266 \text { consumidores online de } 20 \text { a } 34 \text { años de edad } \\
\text { del sector moda de la ciudad de Aguascalientes. }\end{array}$ \\
\hline Sujeto de estudio & $\begin{array}{l}\text { Consumidores online de } 20 \text { a } 34 \text { años de edad del } \\
\text { sector moda de la ciudad de Aguascalientes. }\end{array}$ \\
\hline Muestra & 440 personas \\
\hline Prueba piloto & Aplicación del instrumento a 10 consumidores. \\
\hline $\begin{array}{l}\text { Investigación } \\
\text { cuantitativa }\end{array}$ & $\begin{array}{l}\text { Aplicación del instrumento conformado por escalas } \\
\text { de medición tipo Likert de cinco puntos y una sección } \\
\text { de información general. }\end{array}$ \\
\hline $\begin{array}{l}\text { Recolección de datos y } \\
\text { trabajo de campo }\end{array}$ & $\begin{array}{l}\text { Encuesta electrónica aplicada en el mes de } \\
\text { septiembre de } 2019 \text {. }\end{array}$ \\
\hline Análisis de datos & $\begin{array}{l}\text { Se utilizó el software SPSS } 23.0 \text { y EQS } 6.4 \text {. } \\
\text { Se realizaron análisis descriptivos de la muestra } \\
\text { utilizada. } \\
\text { También se realizó un análisis factorial confirmatorio } \\
\text { para efectuar la comprobación de la fiabilidad y } \\
\text { validez del instrumento utilizado. } \\
\text { Y un modelaje a partir de la técnica de ecuaciones } \\
\text { estructurales para efectuar la contrastación de las } \\
\text { hipótesis de investigación. }\end{array}$ \\
\hline
\end{tabular}

Fuente: Elaboración propia 
Tabla 2. Ítems Experiencia de compra online

Me considero conocedor de buenas técnicas de búsqueda para comprar en este sitio web.

Soy extremadamente hábil para comprar en este sitio web

Sé cómo encontrar lo que estoy buscando cuando hago compras en este sitio web.

Sé algo más que la mayoría de los usuarios sobre las compras en este sitio web.

El uso de este sitio web me reta a realizar mi trabajo lo mejor que pueda.

Considero que el uso de este sitio web extiende mis capacidades a mis límites.

Usar este sitio web me desafía.

Usar este sitio web es una buena prueba de mis habilidades.

El uso de este sitio web crea un mundo nuevo para mí, y este mundo de repente desaparece cuando dejo de navegar.

Me olvido de mi entorno inmediato cuando uso este sitio web.

Este sitio web a menudo me hace olvidar dónde estoy.

Después de comprar en este sitio web, siento que vuelvo al "mundo real" después de un viaje.

Las páginas en este sitio web generalmente se cargan rápidamente.

Interactuar con este sitio web es rápido.

Cuando uso este sitio web, hay poco tiempo de espera entre mis acciones y la respuesta del sitio web.

Es una ventaja cuando el contenido de este sitio web está parcialmente influenciado por la comunidad que lo usa.

Poder conectarse con otros consumidores que comparten intereses similares en el mismo producto es una característica positiva de este sitio web.

Poder compartir comentarios sobre mis experiencias de los productos con otros consumidores en este sitio web es una característica importante para mí.

Ver las recomendaciones de productos de otros consumidores que usan este sitio web es útil.

Este sitio web debería sentir que me está hablando personalmente como cliente.

El requisito de iniciar sesión en este sitio web me hace sentir reconocido como cliente.

Es importante para mí que este sitio web se sienta como mi área personal cuando lo uso.

Me gusta cuando puedo personalizar este sitio web a mi gusto.

La navegación es rápida y fácil cuando compro en este sitio web.

Este sitio web me permite comprar fácilmente lo que quiero.

Es fácil confiar en este sitio web.

Este sitio web es fácil de usar.

Aprender a navegar en este sitio web no me lleva demasiado tiempo.

Siento el control de lo que estoy haciendo cuando compro en este sitio web.

Puedo controlar fácilmente la información que se proporciona en este sitio web.

Siento que puedo controlar el uso que hago de la información en este sitio web.

El nivel de información proporcionado por el sitio web me ayuda a sentir control de mi decisión de compra.

La estética de este sitio web promueve una percepción de calidad.

La marca de este sitio web debe ser coherente con mi actual percepción de esta empresa.

Demasiada publicidad de terceros no es útil cuando se realizan compras en este sitio web.

La apariencia del sitio web es importante cuando se realizan compras.

Puedo aprender qué productos son adecuados para mis necesidades en comparación con 
otros productos competidores navegando por este sitio web.

Con este sitio web puedo averiguar lo que quiero saber antes de comprar en línea.

Al revisar la información provista por este sitio web, puedo estar seguro que tomé la mejor decisión de compra.

La conveniencia de comprar en este sitio web es un beneficio clave.

Al comprar en este sitio web me he sentido totalmente concentrado en lo que estaba haciendo.

Al comprar en este sitio web he perdido la noción del tiempo.

Mientras compraba en este sitio web estaba completamente inmerso en la experiencia de compra.

Mientras compraba en este sitio web me he olvidado de todo lo demás.

Infeliz

Descontento/a

Molesto/a

Triste

Desilusionado/a

Aburrido/a

Decaído/a

Exaltado/a

Desinteresado/a

Nervioso/a

Pasivo/a

Indiferente

Fuente: Elaboración propia

Tabla 3: Ítems Engagement Online

Este sitio web me inspira en mi propia vida.

Este sitio web me hace pensar en cosas de manera innovadora.

Este sitio web estimula mi pensamiento sobre muchos temas diferentes.

Este sitio web me hace una persona más interesante.

Algunas historias en este sitio web me tocan profundamente.

Menciono cosas que he visto en este sitio web en conversaciones con muchas otras personas.

Este sitio web a menudo me da algo de qué hablar.

Uso argumentos de este sitio web en discusiones o intercambios de opiniones con personas que conozco.

Es parte de mi rutina.

Este es uno de los sitios web a los que voy siempre que estoy navegando por la web.

Lo uso como una parte importante para recibir mis noticias del día.

Me ayuda a comenzar mi día en la mañana.

Usar este sitio web me hace sentir como un mejor ciudadano.

El uso de este sitio web hace una diferencia en mi vida.

Este sitio web refleja mis valores.

Me hace más parte de mi comunidad.

Soy una mejor persona cuando uso este sitio web. 
Visitar este sitio web es un placer para mí.

Ir a este sitio web mejora mi estado de ánimo, me hace más feliz.

Me gusta navegar y relajarme en este sitio web.

Me gusta ir a este sitio web cuando estoy comiendo o tomando un descanso.

Mientras estoy en este sitio web, no pienso en otros sitios a los que podría ir.

Este sitio web me ayuda a tomar buenas decisiones de compra.

Aprendes cómo mejorar tus decisiones en este sitio web.

Este sitio web proporciona información que me ayuda a tomar decisiones importantes.

Este sitio web me ayuda a administrar mejor mi dinero.

Doy consejos y sugerencias a las personas que conozco con base en las cosas que he leído en este sitio web.

Hago bastante socialización en este sitio web.

Contribuyo a la conversación en este sitio web.

A menudo me siento culpable por la cantidad de tiempo que dedico a socializar en este sitio web.

Probablemente debería recortar la cantidad de tiempo que dedico a socializar en este sitio web.

Estoy tan interesado en los comentarios de otros usuarios como en el contenido habitual de este sitio web.

Una gran razón por la que me gusta este sitio web es lo que recibo de otros usuarios.

Este sitio web hace un buen trabajo al hacer que sus visitantes contribuyan o proporcionen comentarios.

Me gustaría conocer a otras personas que visitan regularmente este sitio web.

Me he interesado en cosas que comentan otras personas en este sitio web y que no podría obtener en otros sitios.

En general, los visitantes de este sitio web tienen bastante conocimiento sobre los temas que comentan para que pueda aprender de ellos.

Fuente: Elaboración propia

\section{Resultados}

Una vez obtenidos y analizados los resultados de los estadísticos descriptivos de algunas variables, se puede describir el perfil del consumidor online del sector moda de la ciudad de Aguascalientes.

Así, se define que el género del consumidor es femenino, de entre 20 y 24 años de edad, con una escolaridad de licenciatura o ingeniería y unos ingresos mensuales aproximados entre $\$ 0$ y $\$ 6799.00$. En los últimos seis meses compró de dos a cinco veces, principalmente por comodidad y practicidad, variedad de productos y por las promociones de estos. Prefiere realizar sus compras sola desde un smartphone una vez que se encuentra en su hogar. Principalmente compra ropa en tiendas como Amazon, MercadoLibre y Shein. Invierte en ello un monto de $\$ 201.00$ a $\$ 1000.00$. 


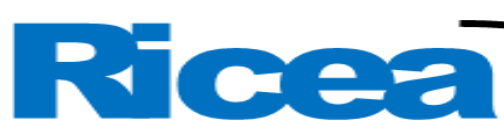

REVISTA IBEROAMERICANA DE CONTADURÍA, ECONOMÍA Y ADMINISTRACIÓN

ISSN $=2007-9907$

Ahora bien, después de realizar las pruebas de fiabilidad y validez de cada una de las escalas utilizadas, y que conforman el modelo teórico propuesto, se presentan los resultados obtenidos de acuerdo con el análisis de sistema de ecuaciones estructurales (SEM), a través del método de máxima verosimilitud, para lo cual se utilizó el software EQS 6.4 y SPSS 23.0. Los resultados obtenidos están dados en función del tratamiento estadístico de la muestra correspondiente a 440 sujetos de investigación.

A continuación, se muestran en la tabla 3 los resultados del análisis estructural en cuanto a los efectos directos entre las variables que conforman el modelo teórico de manera global en correspondencia con la hipótesis planteada en la presente investigación.

Tabla 3. Efectos directos entre las variables que conforman el modelo teórico

\begin{tabular}{|c|c|c|c|c|}
\hline Hipótesis & $\begin{array}{c}\text { Relación } \\
\text { estructural }\end{array}$ & $\begin{array}{c}\text { Coeficiente } \\
\text { estandarizado (B) }\end{array}$ & Valor $\boldsymbol{t}$ & Criterio \\
\hline $\mathrm{H}_{1}$ & $\begin{array}{c}\text { Experiencia de } \\
\text { compra } \text { online } \\
\text { Engagement } \text { online }\end{array}$ & $0.926 * * *$ \\
& & 6.602 & Aceptada \\
$\mathrm{N}=440 ; * * * p<0.01 ; * * p<0.05 ; * p<0.1$ & \\
$=0.837 ; \mathrm{IFI}=0.840 ; \mathrm{GFI}=0.621 ; \mathrm{AGFI}=0.578 ; \mathrm{RMSA}=0.052$. & \\
\hline
\end{tabular}

Fuente: Elaboración propia

De esta manera, se puede establecer una influencia directa y positiva de la experiencia de compra online sobre el engagement online. Como resultado, se obtiene lo siguiente: $\beta=$ 0.926 , con un $p<0.001$, concluyente a partir de un valor de $t=6.602$, el cual es altamente significativo. En otras palabras, la experiencia de compra online generada por los sitios web explica $92.6 \%$ del engagement online de los consumidores del sector moda de la ciudad de Aguascalientes. De acuerdo con los valores anteriores, estos hacen posible aceptar la hipótesis.

En la figura 2 se presenta el modelo plasmado gráficamente con los valores del efecto directo de la variable de acuerdo con la tabla antes mencionada. 


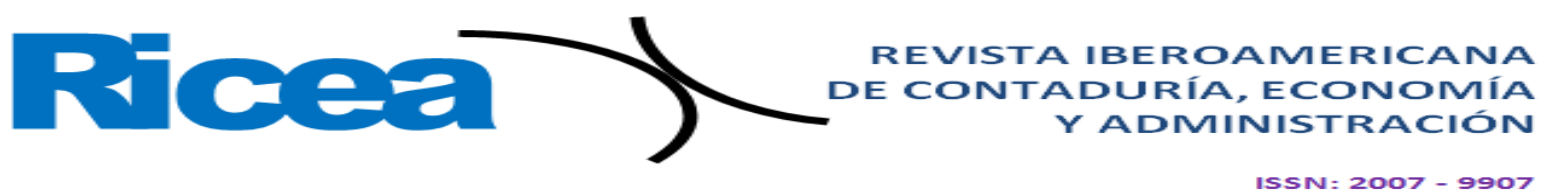

Figura 2. Relación experiencia de compra online-engagement online

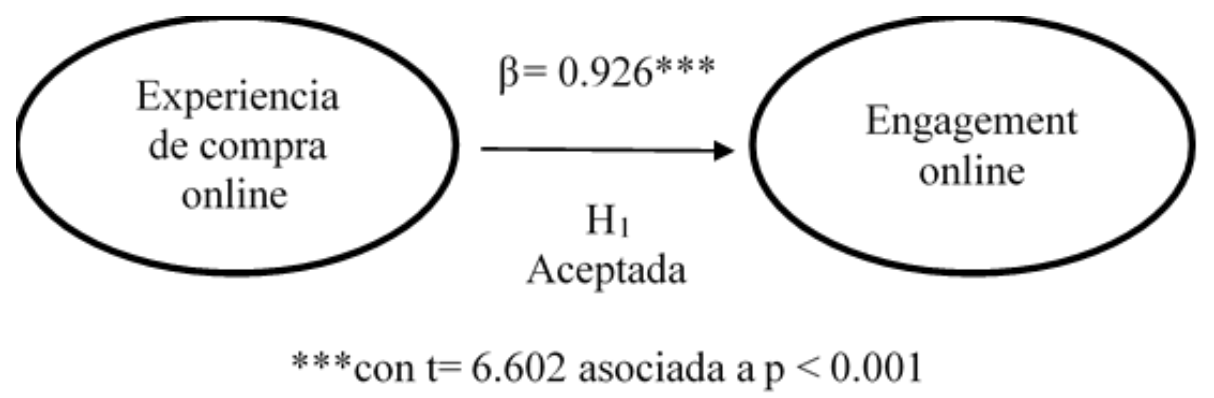

Fuente: Elaboración propia

Otro criterio de evaluación del modelo propuesto es la utilización del coeficiente de determinación $\left(\mathrm{R}^{2}\right)$. De acuerdo con este, existe una relación sustancial entre el engagement online y la experiencia de compra online (0.8574), en donde aquel está explicado en $85.74 \%$ por esta última (ver tabla 4). Al estudiar estos datos, se encuentra suficiente evidencia empírica de la consistencia del modelo planteado respecto a la teoría referida en la presente investigación.

Tabla 4. Resultados $\mathrm{R}^{2}$ obtenidos a partir del análisis estructural del modelo

\begin{tabular}{|c|l|c|}
\hline Variables explicativas & Variable explicada & $\mathbf{R}^{\mathbf{2}}$ \\
\hline Experiencia de compra online & Engagement online & $85.74 \%$ \\
\hline
\end{tabular}

Fuente: Elaboración propia

\section{Discusión}

La experiencia de compra online es un proceso holístico y subjetivo que resulta de las interacciones entre los propios consumidores y el entorno online, ya sea mediante el uso de sitios web de comercio electrónico, opiniones o valoraciones de productos de otros usuarios, redes sociales, entre otros (Trevinal y Stenger, 2014). Los resultados aquí encontrados apoyan esta teoría, ya que la experiencia de compra es individualizada: ninguna va a ser igual a la de otra persona y solo a través de la interacción del consumidor con el sitio web se podrá dar una experiencia de compra online.

Por otra parte, Gentile, Spiller y Noci (2007) afirman que el consumidor interpreta las experiencias de compra desde una perspectiva, por un lado, cognitiva, involucrando pensamientos o procesos mentales conscientes, y por el otro, afectiva, que implica el proceso afectivo del consumidor a través de la generación de estados de ánimo, sentimientos y 


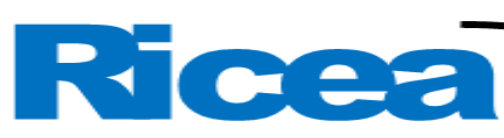

REVISTA IBEROAMERICANA DE CONTADURÍA, ECONOMÍA Y ADMINISTRACIÓN

ISSN $=2007-9907$

emociones (Rose et al., 2012; Rose, Hair y Clark, 2011). Ahora bien, la exposición repetida del consumidor a un sitio web hace que el procesamiento cognitivo y afectivo de la información sensorial emitida por este forme una impresión acumulativa en la memoria con el tiempo, lo que hace establecer que la experiencia de compra online sea resultado de ello (Rose et al., 2012).

Estas aseveraciones están igualmente en concordancia con los resultados obtenidos, pues se pudo corroborar que ambas dimensiones componen el constructo de la experiencia de compra online planteado en la presente investigación, además de que los ítems que integran cada una de ellas hacen referencia a los descritos por la teoría. De hecho, en los últimos años se encuentran varias investigaciones previas que demuestran una relación directa y positiva (Brodie et al., 2013; Cheng, Yang y Chen, 2011; Grissemann y StokburgerSauer, 2012; Gummerus, Liljander, Weman y Pihlstrom, 2012; Ha e Im, 2012; Jaakkola y Alexander, 2014; Ki-Han y Jae-Ik, 2010; Kim y Park, 2013; Klaus, 2013; Kumar et al., 2010; Maklan y Klaus, 2011; O’Cass y Carlsson, 2010; Shobeiri, Mazaheri y Laroche, 2014; Tran, Strutton y Taylor, 2012; Trevinal y Stenger, 2014; Van Doorn et al., 2010; Wei, Miao y Huang, 2013; Won-Moo, Kwang-Ho y Kim, 2011; Wu, Lee, Fu y Wang, 2013).

En este sentido, los resultados obtenidos en este estudio se ajustan a los de algunos autores que han concluido en sus trabajos que los consumidores, después de realizar una compra online, presentan respuestas positivas, tales como: una mayor probabilidad de volver a visitar el sitio web de e-commerce en un futuro (Hausman y Siekpe, 2009; Koufaris, 2002), una mayor intención de compra (Fiore y Kim, 2007; Ha e Im, 2012; Rose et al., 2012) y actos de recomendación o publicidad boca-oreja acerca de dicha experiencia (Cheng et al., 2011; Fiore y Kim, 2007; Ha e Im, 2012; Ki-Han y Jae-Ik, 2010; Kim y Park, 2013; Ladhari, 2007; O’Cass y Carlsson, 2010; Tran et al., 2012; Won-Moo et al., 2011; Wu et al., 2013). Dichas respuestas se presentan de manera intrínseca en algunos conceptos de engagement online (Brodie et al., 2013; Grissemann y Stokburger-Sauer, 2012; Van Doorn et al., 2010).

En cuanto al engagement online, Brodie et al. (2011) lo definen como un vehículo para la creación y mantenimiento de las relaciones entre el consumidor y la empresa caracterizado por ser un estado psicológico que sucede bajo ciertas condiciones que dependen del contexto en el que se desarrollan las experiencias interactivas y cocreativas de los consumidores con una marca, ya sea en un sitio web u otras entidades mediadas por computadora; así, se generan diferentes niveles de compromiso dentro de las relaciones de 


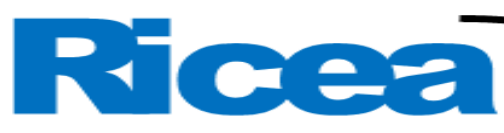

REVISTA IBEROAMERICANA DE CONTADURÍA, ECONOMÍA Y ADMINISTRACIÓN

$1 S S N=2007-9907$

servicio que crean valor más allá de la compra (Hollebeek, 2011; Mollen y Wilson, 2010; Patterson et al., 2006; Van Doorn et al., 2010; Vivek et al., 2012).

Los resultados logrados están en sintonía con la afirmación anterior. Efectivamente, el engagement online es resultado de las diferentes experiencias tanto sensoriales como afectivas, cognitivas, sociales y de comportamiento brindadas al consumidor a través del sitio web de una marca. Asimismo, concuerdan con el hecho de que este es un estado psicológico que crea relaciones de valor que van más allá de la compra, por ejemplo, lograr que un sitio web sea una inspiración para el consumidor, que converse con otras personas acerca del contenido de este, que la navegación en él sea parte de su rutina, que lo relaje y que la experiencia sea placentera, vea reflejados sus valores y se sienta parte de una comunidad, por lo que no piensa en visitar otro sitio web diferente.

Aunado a ello, los resultados son compatibles con los presentados por Kumar et al. (2010), quienes señalan que el engagement es una conexión profunda y significativa entre el consumidor y una marca, perdurable en el tiempo y generada por transacciones como las compras. En este sentido, la experiencia de compra online fue el constructo que más explica al engagement online de acuerdo con los datos del análisis de ecuaciones estructurales.

Los hallazgos encontrados en este estudio indican que las empresas del sector moda deben crear experiencias de compra placenteras a través de la dotación de las diez variables que fungen como antecedentes (habilidad, nivel de desafío, telepresencia, velocidad interactiva, control percibido, conectividad, customización, facilidad de uso, estética, beneficios percibidos) del estado experiencial cognitivo y afectivo para generar un impacto positivo en el engagement online del consumidor de la ciudad de Aguascalientes. Estos resultados coinciden con las investigaciones científicas antes mencionadas. Sin duda hay una relación significativa entre las variables aquí en cuestión.

$\mathrm{Si}$ bien los datos de esta investigación coinciden ampliamente con diversas investigaciones previas, es importante señalar que, al encontrar que la variable de la experiencia de compra online aporta mucho al engagement online, los empresarios inmersos en el comercio electrónico deberán fortalecer esta variable si desean generar engagement con el consumidor.

En consideración tanto a las definiciones como a los resultados obtenidos en la presente investigación, se puede demostrar que la $\mathrm{H}_{1}$ se acepta. Es decir, $\mathrm{H}_{1}$ : la experiencia de compra online influye de manera directa y positiva en el engagement online del 


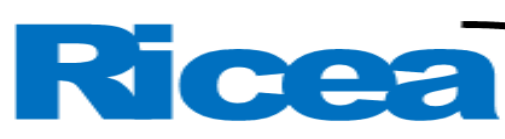

REVISTA IBEROAMERICANA DE CONTADURÍA, ECONOMÍA Y ADMINISTRACIÓN

consumidor del sector moda de la Ciudad de Aguascalientes.

Por otro lado, hay que señalar como principales limitaciones del estudio que el sector en el que se desarrolló este fue únicamente en el de la moda. Como ya es sabido, se enfocó en analizar la última experiencia de compra online del participante en los últimos seis meses, lo que no refleja un comportamiento certero de este, ya que probablemente intervinieron factores como el tiempo transcurrido, la falta de claridad en sus emociones, poca motivación y concentración al momento de contestar la encuesta que influyeron en su opinión. Respecto a esto último, se considera que la extensión del cuestionario puede ser un factor para desalentar la objetividad de las respuestas y sesgar la información.

La muestra comprendió individuos de 20 a 34 años de edad de la ciudad de Aguascalientes, México, lo que igualmente limita la generalización de los resultados a sujetos de otros segmentos de la población. Además, el muestreo por bola de nieve puede provocar un sesgo en los datos y tener una muestra no representativa.

Como resultado de la presente investigación, se identifican algunas futuras líneas de investigación que podrían replicar el modelo propuesto en un contexto de compra de bienes de carácter utilitario, como electrodomésticos o de salud; o bienes intangibles como servicios de mensajería y paquetería, educativos, financieros o gubernamentales. Lo anterior podría llevar a realizar un análisis comparativo de diversos sectores empresariales que lleve a generalizar, ahora sí, el comportamiento del consumidor online.

Por otro lado, para analizar el comportamiento real del consumidor es imprescindible que la investigación empírica se realice mediante instrumentos incorporados directamente a los sitios web para que estos sean contestados por el usuario inmediatamente después de haber vivido las experiencias, sin que estas sean afectadas por otras variables.

Como otra alternativa, vale la pena mencionar que en las futuras investigaciones, además del instrumento aplicado, el consumidor pudiera someterse a técnicas de neuromarketing para contrastar sus respuestas textuales con aquellas que se encuentran de manera inconsciente en el individuo y así medir sus emociones de manera más precisa. 


\section{Conclusiones}

A partir de los resultados obtenidos, se pueden realizar propuestas prácticas a aquellas empresas que ya efectúen o quieran realizar e-commerce de artículos de moda, y también a todas aquellas empresas que a través de un sitio web vendan sus productos, independientemente del giro en el que se encuentren. Dichas recomendaciones tendrán como fin el mejoramiento del desempeño de las organizaciones y la obtención de ventajas competitivas sostenibles a largo plazo en cuanto a la ejecución de estrategias de experiencia de compra online y engagement online.

En este sentido, las organizaciones deben procurar un estado de "flujo" en el que el consumidor mentalmente se encuentre inmerso por el placer y goce que le ocasiona realizar una compra online: generarle una pérdida de la noción del tiempo, espacio y otras cosas que pasen a su alrededor. En razón de lo anterior, los sitios web deberán fomentar la capacidad del consumidor para navegar e interactuar con este al momento de realizar el proceso de compra. De igual manera, el sitio web debe provocar en el usuario un nivel de desafío para que no le parezca aburrido navegar ahí, es decir, que lo exhorte a poner a prueba sus habilidades.

También es importante mencionar que lo más esencial para fortalecer la experiencia de compra online es que el consumidor perciba el entorno online como real, como si estuviera en un espacio físico de compra; crear un mundo alterno al real capaz de provocar en el internauta que se olvide incluso de su entorno inmediato. Asimismo, garantizar un tiempo de respuesta inmediato del sitio web hacia las acciones del usuario permite experimentar mejor la experiencia de compra online, ya que hoy en día los usuarios buscan la inmediatez y prontitud en un entorno virtual.

Como complemento a lo anterior, el consumidor hoy en día busca tener control sobre el sitio web de su preferencia; promueve que un sitio web sea capaz de permitirle compartir ideas y experiencias de consumo con otros usuarios que presentan intereses similares, e incluso contribuir en la generación del propio contenido del sitio web.

De igual manera, es muy valorado por los individuos que un sitio web esté personalizado de acuerdo con sus necesidades, ya que, al adaptar la información y el contenido de este, se siente como si le hablara específicamente al usuario. Esto se puede lograr desde el momento en que el visitante se registra, crea una cuenta y un perfil, es decir, es reconocido por la marca al proporcionarle un área personal diseñada también a su gusto. 


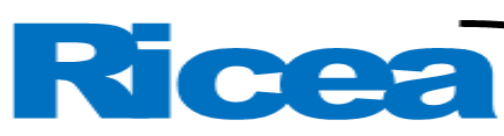

REVISTA IBEROAMERICANA DE CONTADURÍA, ECONOMÍA Y ADMINISTRACIÓN

ISSN $=2007-9907$

Indiscutiblemente, en la medida en que la navegación, la búsqueda de información y funcionalidad del sitio web sean favorables para los usuarios, se mejorará la experiencia de compra online, por lo que las empresas deberán procurar crear sitios web intuitivos, sin tantas ventanas que hagan que el consumidor se pierda, diseñar menús claros con la información más relevante a primera vista, clarificar el proceso de compra, generar confianza en la transacción mediante políticas de compras muy bien definidas o a través de mecanismos de pago ya garantizados.

Parte esencial de crear una mejor experiencia es cuidar la estética del entorno virtual, por lo que las marcas deberán tener cuidado en algunos factores como el color utilizado, tanto en los textos como de fondo, las características de los gráficos, especialmente la calidad de las imágenes, el diseño multimedia y disposición de los contenidos, los cuales, en conjunto, hacen que el diseño de la interfaz del sitio web invite al consumidor a disfrutar la navegación, a comprar y a satisfacer sus necesidades. También, el consumidor manifiesta que demasiada publicidad no es útil cuando se realizan las compras, pues demerita la calidad del sitio web.

El usuario debe percibir que recibe beneficios al navegar y comprar en determinado sitio web. Dichos beneficios pueden estar en la opción de poder comparar precios entre los diferentes productos que se ofertan, la conveniencia de comprarlo por ahorro económico, de tiempo, facilidad de la transacción o servicios posventa, así como la diversión proporcionada al momento de comprar. De igual manera, el consumidor valora que en el sitio web se le administre una orientación acerca de qué productos realmente satisfarán sus necesidades, lo cual se puede lograr mediante una reseña de las características del artículo, exposición de una serie de consejos para su uso en la que se resalten algunas ventajas o desventajas y la elaboración de un diagnóstico de sus necesidades mediante un formulario. Todo lo anterior llevará al usuario a clarificar si cierto producto cubre sus necesidades antes de realizar la compra.

Además de fortalecer estas experiencias, no está de más remarcar que las marcas en sus sitios web deben generar un entorno en el que el consumidor crea que el contexto online es su realidad, es decir, se olvide del mundo offline y navegue sin preocupaciones en el sitio web con la finalidad de mejorar la experiencia de compra online. Una de las alternativas que podrían implementar en esta implicación sería mediante la aplicación de sistemas de realidad virtual, visitas 3D, chats o foros de conversación en tiempo real con el usuario y entre ellos. 


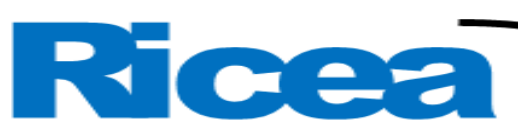

REVISTA IBEROAMERICANA DE CONTADURÍA, ECONOMÍA Y ADMINISTRACIÓN

Aunado a lo anterior, deberá ejecutar estrategias y tácticas que permitan desafiar las capacidades del usuario, en otras palabras, que la navegación en el sitio web, cuando está realizando el proceso de compra, no se le haga aburrida, tediosa o sin novedad. Para tal efecto, las marcas pueden considerar incorporar en sus sitios web estrategias de gaming marketing, táctica de marketing digital en la que, además de mejorar la imagen de la marca, se incrementa la base de datos de los clientes y se crean comunidades. Esto último también con el propósito de brindar las experiencias sociales que busca el consumidor y que fueron mencionadas con anterioridad. Dicha implicación adquiere relevancia porque, al llevar al límite sus habilidades y motivar al consumidor para que continúe navegando o concluya su compra, se podría incrementar la experiencia de compra online.

Por lo tanto, las empresas deben procurar la dotación de los elementos antes mencionados en un sitio web para mejorar la experiencia de compra online. Con esto se podrá suscitar un engagement online integrado por un engagement personal y uno social-interactivo. El primero se logrará en la medida de que el sitio web sea innovador, inspire al usuario a hacer cosas diferentes o de manera desigual y su contenido lo toque en lo más profundo. De igual manera, se puede manifestar cuando el usuario menciona en sus conversaciones cosas que ha visto en el sitio web; en suma, que sea un tema de qué hablar o una caja de argumentos a la hora de intercambiar opiniones.

Si se logra una eficiente experiencia de compra online también se puede lograr un engagement personal a través de la navegación recurrente en el sitio web como algo rutinario y esencial, de tal manera que contribuya en el usuario a sentirse una mejor persona y parte de una comunidad. Esta última reacción del consumidor ante una buena experiencia de compra online es la que tiene mayor relación con este constructo, es decir, que la experiencia de compra online impacte fuertemente en la autoestima y civismo del individuo.

Por otro lado, para lograr un engagement social-interactivo mediante la experiencia de compra online es necesario alcanzar cuatro aspectos importantes: el disfrute intrínseco, utilitario, participación y socialización y, por último, comunidad. El primero se manifiesta cuando el consumidor presenta placer al realizar la compra en el sitio web, mejora su estado de ánimo e incluso presenta felicidad, según lo manifestado, se siente relajado, lo visita cuando tiene espacios de tiempo (horas de comida y ocio) y no piensa en visitar otros. El segundo, es observado cuando el usuario percibe que el sitio web le ayuda a tomar buenas decisiones de compra, a administrar mejor su dinero, e incluso le permite orientar a otras 


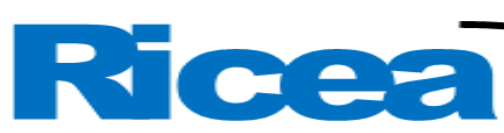

REVISTA IBEROAMERICANA DE CONTADURÍA, ECONOMÍA Y ADMINISTRACIÓN

personas acerca de sus compras.

El siguiente elemento se logra cuando el consumidor socializa en el sitio web, conversa y siente que pasa mucho tiempo en él, al grado de creer que debe reducir su tiempo de navegación. El último de estos aspectos está vinculado con el interés del usuario por los comentarios que puede recibir de otros integrantes de la comunidad, incluso de que quiera conocer a algunos personalmente porque siente que aprende de ellos, así como del contenido del sitio web porque considera que tanto este como las aportaciones de otros usuarios no las pudiera obtener de otro sitio.

También es importante para las empresas que el sitio web se asegure de reflejar los valores del consumidor y lo hagan sentirse mejor ser humano, por lo que deberán cuidar que todos los estímulos visuales y auditivos que se puedan brindar al consumidor mantengan una concordancia con la filosofía del cliente, de lo contrario, no generarán engagement con este. Si el consumidor siente que el sitio web le está aportando a su crecimiento como persona, entonces tendrá más confianza en él para visitarlo y comprar. Esto se podrá lograr si las organizaciones tienen un conocimiento profundo del perfil de su mercado.

No hay que olvidar que deberán incrementar sus estrategias para que el consumidor tenga interés en visitar la página web de comercio electrónico sobre todas las cosas, que comience su día visitándolo, reciba noticias de este y lo haga parte de su día a día, con la intención de que a través de ello se pueda generar un engagement online. Para poder alcanzar lo anterior, es necesario que las empresas realicen investigaciones de mercado y hagan uso de la minería de datos para que detecten las necesidades del consumidor y, además, mediante estrategias de marketing digital y marketing directo, le hagan llegar noticias, consejos, promociones e incluso diseñar planes de fidelización al potencial comprador.

Por último, en cumplimiento con el objetivo del estudio, se valida la relación entre la experiencia de compra online y el engagement online, pues existe evidencia que la experiencia de compra online ejerce una influencia positiva, directa y significativa sobre el engagement online del consumidor. 


\section{Referencias}

Asociación Mexicana de Venta Online [AMVO], Elogia y Netquest. (2018). Estudio Comercio Electrónico en Moda 2018. México: Asociación Mexicana de Venta Online, Elogia y Netquest. Recuperado de https://docs.wixstatic.com/ugd/5e9e8f_802fba75289b4d758fd8ff42b0063782.pdf.

Blázquez, M. (2014). Fashion shopping in multichannel retail: The role of technology in enhancing the customer experience. International Journal of Electronic Commerce, 18(4), 97-116.

Brodie, R. J., Hollebeek, L. D., Juric, B. and Ilic, A. (2011). Customer engagement: Conceptual domain, fundamental propositions, and implications for research. Journal of Service Research, 14(3), 252-271.

Brodie, R. J., Ilic, A., Juric, B. and Hollebeek, L. (2013). Consumer engagement in a virtual brand community: An exploratory analysis. Journal of Business Research, 66(1), 105-114. Retrieved from http://doi:10.1016/j.jbusres.2011.07.029.

Calder, B. J., Malthouse, E. C. and Schaedel, U. (2009). An Experimental Study of the Relationship between Online Engagement and Advertising Effectiveness. Journal of Interactive Marketing, 23(4), 321-331.

Cheng, H., Yang, M. and Chen, K. (2011). Elucidating the ethical sales behavior in electronic commerce. The Journal of Computer Information Systems, 52(1), 87-95.

comScore. (2017). Estudio sobre la Experiencia del Comprador. Resumen de resultados. Septiembre 2017. México: comScore. Recuperado de https://docs.wixstatic.com/ugd/5e9e8f_1783be29f4884b53a6800caff6e0ae3d.pdf.

Fiore, A. M. and Kim, J. (2007). An integrative framework capturing experiential and utilitarian shopping experience. International Journal of Retail \& Distribution Management, 35(6), 421-442.

Gentile, C., Spiller, N. and Noci, G. (2007). How to Sustain the Customer Experience: An Overview of Experience Components that Co-create Value with the Customer. European Management Journal, 25(5), 395-410.

Grewal, D., Levy, M. and Kumar, V. (2009). Customer Experience Management in Retailing: An organizing framework. Journal of Retailing, 85(1), 1-14.

Grissemann, U. S. and Stokburger-Sauer, N. E. (2012). Customer co-creation of travel services: The role of company support and customer satisfaction with the co-creation 


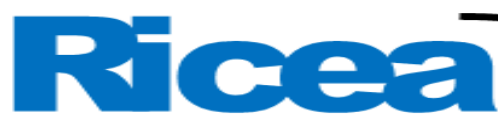

REVISTA IBEROAMERICANA DE CONTADURÍA, ECONOMÍA Y ADMINISTRACIÓN

performance. Tourism Management, 33(6), 1483-1492.

Gummerus, J., Liljander, V., Weman, E. and Pihlstrom, M. (2012). Customer engagement in a Facebook brand community. Management Research Review, 35(9), 857-877.

Ha, Y. and Im, H. (2012). Role of web site design quality in satisfaction and word of mouth generation. Journal of Service Management, 23(1), 79-96.

Hausman, A. and Siekpe, J. (2009). The effect of web interface features on consumer online purchase intentions. Journal of Business Research, 62(1), 5-13.

Hollebeek, L. D. (2011). Demystifying customer brand engagement: exploring the loyalty nexus. Journal of Marketing Management, 27(7-8), 785-807.

Instituto Nacional de Estadística y Geografía [Inegi]. (2018). Estadísticas a propósito de... la industria de la moda del vestido, el calzado y la joyería. Aguascalientes, México: Instituto Nacional de Estadística y Geografía. Recuperado de http://internet.contenidos.inegi.org.mx/contenidos/Productos/prod_serv/contenidos/ espanol/bvinegi/productos/estudios/economico/a_proposi_de/EstModaCalzaJoyeria _18.pdf.

Irala, C. (2 de enero 2018). Comercio electrónico: estadísticas del 2017 y tendencias para el 2018. Latamclick. Recuperado de https://www.latamclick.com/estadisticascomercio-electronico-2017-tendencias-2018/.

Jaakkola, E. and Alexander, M. (2014). The role of customer engagement behavior in value co-creation: a service system perspective. Journal of Service Research, 17(3), 247261.

Ki-Han, C. and Jae-Ik, S. (2010). The antecedents and consequents of relationship quality internet shopping. Asia Pacific Journal of Marketing and Logistics, 22(4), 473-491.

Kim, S. and Park, H. (2013). Effects of various characteristics of social commerce (scommerce) on consumers' trust and trust performance. International Journal of Information Management, 33(2), 318-332.

Klaus, P. (2013). The case of Amazon. com: towards a conceptual framework of online customer service experience (OCSE) using the emerging consensus technique (ECT). Journal of Services Marketing, 27(6), 443-457.

Koufaris, M. (2002). Applying the technology acceptance model and flow theory to online consumer behavior. Information Systems Research, 13(2), 205-223.

Kumar, V., Aksoy, L., Donkers, B., Venkatesan, R., Wiesel, T. and Tillmanns, S. (2010). 
Undervalued or overvalued customers: capturing total customer engagement value. Journal of Service Research, 13(3), 297-310.

Ladhari, R. (2007). The effect of consumption emotions on satisfaction and word-of-mouth communications. Psychology \& Marketing, 24(12), 1085-1108.

López, E. (2017). Marketing experiencial: una aplicación a la experiencia de compra online en el sector moda. (tesis de doctorado). Universidad Complutense de Madrid, España. Recuperado de https://eprints.ucm.es/40809/1/T38243.pdf.

Maklan, S. and Klaus, P. (2011). Customer experience: are we measuring the right things? International Journal of Market Research, 53(6), 771-792.

Mollen, A. and Wilson, H. (2010). Engagement, telepresence and interactivity in online consumer experience: Reconciling scholastic and managerial perspectives. Journal of Business Research, 63(9-10), 919-925.

O’Cass, A. and Carlson, J. (2010). Examining the effects of website-induced flow in professional sporting team websites. Internet Research, 20(2), 115-134.

Patterson, P., Yu, T. and de Ruyter, K. (2006). Understanding Customer Engagement in Services. Paper presented at the 2006 Australian and New Zealand Marketing Academy Conference. Brisbane, December 4-6, 2006.

Rose, S., Clark, M., Samouel, P. and Hair, N. (2012). Online Customer Experience in eRetailing: An empirical model of Antecedents and Outcomes. Journal of Retailing, 88(2), 308-322.

Rose, S., Hair, N. and Clark, M. (2011). Online Customer Experience: A Review of the Business-to-Consumer Online Purchase Context. International Journal of Management Reviews, 13(1), 24-39.

Shobeiri, S., Mazaheri, E. and Laroche, M. (2014). Improving customer website involvement through experiential marketing. The Service Industries Journal, 34(11), 885-900.

Tran, G. A., Strutton, D. and Taylor, D. G. (2012). Do microblog postings influence consumer perceptions of retailers' e-servicescapes? Management Research Review, 35(9), 818-836.

Trevinal, A. M. and Stenger, T. (2014). Toward a conceptualization of the online shopping experience. Journal of Retailing and Consumer Services, 21(3), 314-326.

Van Doorn, J., Lemon, K. N., Mittal, V., Nass, S., Pick, D., Pirner, P. and Verhoef, P. C. (2010). Customer engagement behavior: Theoretical foundations and research 


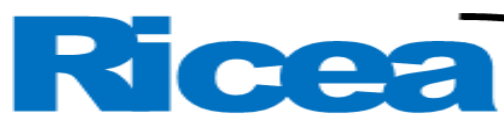

REVISTA IBEROAMERICANA

DE CONTADURÍA, ECONOMÍA

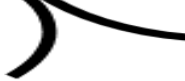

YADMINISTRACIÓN

directions. Journal of Service Research, 13(3), 253-266.

Vivek, S. D., Beatty, S. E. and Morgan, R. M. (2012). Customer engagement: Exploring customer relationships beyond purchase. Journal of Marketing Theory and Practice, 20(2), 127-145.

Wei, W., Miao, L. and Huang, Z. J. (2013). Customer engagement behaviors and hotel responses. International Journal of Hospitality Management, 33(1), 316-330.

Won-Moo, H., Kwang-Ho, A. and Kim, A. (2011). Building brand loyalty through managing brand community commitment. Management Decision, 49(7), 1194-1213.

Wu, W. Y., Lee, C. L., Fu, C. S. and Wang, H. C. (2013). How can online store layout design and atmosphere influence consumer shopping intention on a website? International Journal of Retail \& Distribution Management, 42(1), 4-24. 


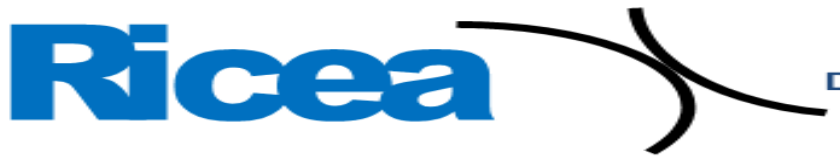

\begin{tabular}{|c|c|}
\hline Rol de Contribución & Autor (es) \\
\hline Conceptualización & $\begin{array}{l}\text { Vianney Judith Robledo Herrera.- principal } \\
\text { Elena Patricia Mojica Carrillo.- igual }\end{array}$ \\
\hline Metodología & Elena Patricia Mojica Carrillo.- principal \\
\hline Software & $\begin{array}{l}\text { Vianney Judith Robledo Herrera.- principal } \\
\text { Elena Patricia Mojica Carrillo.- igual }\end{array}$ \\
\hline Validación & $\begin{array}{l}\text { Vianney Judith Robledo Herrera.- principal } \\
\text { Elena Patricia Mojica Carrillo.- igual }\end{array}$ \\
\hline Análisis Formal & $\begin{array}{l}\text { Vianney Judith Robledo Herrera.- principal } \\
\text { Elena Patricia Mojica Carrillo.- igual }\end{array}$ \\
\hline Investigación & Vianney Judith Robledo Herrera.- principal \\
\hline Recursos & Vianney Judith Robledo Herrera.- principal \\
\hline Curación de datos & Vianney Judith Robledo Herrera.- principal \\
\hline $\begin{array}{l}\text { Escritura - Preparación del } \\
\text { borrador original }\end{array}$ & $\begin{array}{l}\text { Sofía Elba Vázquez Herrera.- principal } \\
\text { Fernando Rey Castillo Villar.- apoyo }\end{array}$ \\
\hline $\begin{array}{l}\text { Escritura - Revisión } \\
\text { edición }\end{array}$ & $\begin{array}{l}\text { Sofía Elba Vázquez Herrera.- principal } \\
\text { Fernando Rey Castillo Villar.- apoyo }\end{array}$ \\
\hline Visualización & $\begin{array}{l}\text { Sofía Elba Vázquez Herrera.- principal } \\
\text { Fernando Rey Castillo Villar.- apoyo }\end{array}$ \\
\hline Supervisión & $\begin{array}{l}\text { Vianney Judith Robledo Herrera.- principal } \\
\text { Elena Patricia Mojica Carrillo.- igual }\end{array}$ \\
\hline $\begin{array}{l}\text { Administración } \quad \text { de } \\
\text { Proyectos }\end{array}$ & $\begin{array}{l}\text { Vianney Judith Robledo Herrera.- principal } \\
\text { Elena Patricia Mojica Carrillo.- igual }\end{array}$ \\
\hline Adquisición de fondos & Elena Patricia Mojica Carrillo.- principal \\
\hline
\end{tabular}

